\title{
INFLUÊNCIA DAS CARACTERÍSTICAS DAS SUBSTÂNCIAS HÚMICAS NA EFICIÊNCIA DA COAGULAÇÃO COM SULFATO DE ALUMÍNIO
}

\author{
THE INFLUENCE OF HUMIC SUBSTANCES CHARACTERISTICS ON THE \\ COAGULATION EFFICIENCY USING ALUMINUM SULFATE
}

\author{
SANDRO XAVIER CAMPOS \\ Químico e Doutor em Engenharia Civil - Área de Concentração: Hidráulica e Saneamento. \\ Escola de Engenharia de São Carlos - USP

\section{LUIZ DI BERNARDO} \\ Engenheiro Civil. Doutor em Engenharia Civil da Escola de Engenharia de São Carlos - USP.
}

Professor Titular da Escola de Engenharia de São Carlos - USP

\section{ENY M. VIEIRA}

Química. Professora do Instituto de Química de São Carlos - USP

Recebido: 18/11/04 Aceito: 17/05/05

\section{RESUMO}

Neste trabalho foi estudada a influência das características das substâncias húmicas $(\mathrm{SH})$, em especial a massa molar das moléculas, na eficiência da coagulação com o uso do sulfato de alumínio. Utilizando-se de água proveniente de poço profundo, foram preparadas quatro águas de estudo, todas com cor verdadeira de aproximadamente $100 \mathrm{uH}$. As $\mathrm{SH}$ foram extraídas de turfa e fracionadas em massas molares: i) $\mathrm{SH}$ filtradas em membranas de $0,45 \mu \mathrm{m}$; ii) $\mathrm{SH}$ filtradas em membranas de $0,45 \mu \mathrm{m}$ e maiores que $100 \mathrm{kDa}$, iii) SH com moléculas entre 30 e $100 \mathrm{kDa}$; iv) SH com moléculas menores do que $30 \mathrm{kDa}$. Verificou-se que quanto maior a massa molar das $\mathrm{SH}$ maior foi a eficiência de remoção da cor aparente, com as menores dosagens de coagulante. $\mathrm{O} \mathrm{pH}$ de coagulação ótimo, esteve entre 6,0 e 7,0, para todas as águas de estudo. Os estudos da porcentagem de ácido húmico $(\mathrm{AH})$ e ácido fúlvico (AF) presentes em cada fração de diferente massa molar mostraram que quanto maior foi à porcentagem de $\mathrm{AF}$ presente, maior foi à dosagem de coagulante exigida para se obter os melhores resultados de remoção da cor aparente.

PALAVRAS-CHAVE: Substâncias húmicas, massa molecular das substâncias húmicas, ácido húmico, ácido fúlvico, coagulação com sulfato de alumínio.

\begin{abstract}
The influence of the molecular weight of soil humic substances on the coagulation efficiency with aluminum sulfate was studied in this work. Four types of water were studied, with the same true color of approximately $100 \mathrm{HU}$, but prepared with different fractions of molecular weights: Water Type 1 (humic substances filtered in membrane of 0,45 $\mu \mathrm{m}$ ); Water Type 2 (humic substances filtered in membrane of 0,45 $\mu \mathrm{m}$ and with molecular weight $>100 \mathrm{KDa}$ ); Water Type 3 (humic substances with molecular weight between 100 and $30 \mathrm{KDa}$ ); Water Type 4 (humic substances with molecular weight $<30 \mathrm{KDa}$ ). It was conclude that the higher the molecular weight of the molecules, the lower the coagulant dosages required for efficient coagulation, at the coagulation $p H$ range $6-7$. The types of water prepared with the fractions of humic substances containing higher concentrations offulvic acid required higher doses of coagulant to achieve the same efficiency.
\end{abstract}

KEYWORDS: Humic substances, humic acid, fulvic acid, molecular weight, coagulation with aluminum sulfate.

\section{INTRODUÇÃO}

A água natural contém substâncias que variam amplamente em sua origem, concentração e características. O tamanho das partículas pode ser de diversas ordens de grandeza. Os vírus atingem cerca de $10^{-6} \mathrm{~mm}$, as partículas coloidais encontram-se entre $10^{-6}$ e $10^{-3} \mathrm{~mm}$, o zooplâncton chega a $10^{-2} \mathrm{~mm}$ e as moléculas de substâncias húmicas ( $\mathrm{SH})$, dependendo do grau de polimerização, po- dem atingir valores entre 3,5 e $10 \times 10^{-6} \mathrm{~mm}$ (AWWA, 1990).

As partículas possuem cargas elétricas negativas em sua superfície, que criam uma barreira repulsiva entre si e impossibilita sua aglomeração. Desta forma, torna-se necessário promover a alteração das características da superfície das partículas através da adição de coagulantes, geralmente sais de alumínio ou de ferro. Quando o alumínio é adicionado na água e hidrolisa, há a formação de um grande número de espécies monoméricas, e possíveis espécies poliméricas. A maior parte desses produtos encontra-se em equilíbrio com o precipitado sólido de hidróxido de alumínio $\left[\mathrm{Al}(\mathrm{OH})_{3}\right]$. Estas espécies hidrolisadas podem ainda incluir compostos de alumínio polinucleares. A natureza dos produtos formados é afetada pelo $\mathrm{pH}$ de origem da água, alcalinidade, temperatura, outras partículas, matéria orgânica, dosagem de coagulante e $\mathrm{pH}$ de coagulação (Amirtharajah \& O’Melia, 1990). 
A coagulação e a floculação são etapas de grande importância pois possuem relação direta com o desempenho da sedimentação e filtração. A eficiência da coagulação e da floculação é influenciada por fatores como $\mathrm{pH}$, turbidez, alcalinidade, dosagem de coagulante, intensidade de agitação, ponto de aplicação do coagulante, tamanho e distribuição do tamanho das partículas, sólidos totais dissolvidos, cor, etc (Di Bernardo et al, 2002). Sendo a coagulação vastamente empregada no tratamento de água, uma ferramenta que seja capaz de predizer qual $\mathrm{pH}$ mais efetivo para que a coagulação ocorra e a dosagem de coagulante necessária, é extremamente útil (Kim et al, 2001). Edwards \& Amirtharajah (1985) desenvolveram o diagrama de coagulação com alumínio para remoção de cor, útil para planejar, analisar e interpretar resultados obtidos na coagulação em equipamentos de jarteste e em instalação piloto.

Para o estudo de remoção de cor, as SH são amplamente empregadas para preparar águas de estudo visando a realização de ensaios de coagulação em equipamento de jarteste. A justificativa em seu uso pode ser encontrada no fato de que cerca de 50 a $70 \%$ de todo carbono dissolvido em águas naturais não poluídas, seja devido a sua presença (Aiken, 1985). As SH empregadas em estudos de coagulação podem ser originadas de diferentes matrizes (solo, turfa e aquática) o que torna essencial a determinação de algumas de suas principais características para que os resultados possam ser comparados com outros estudos.

Desta forma este trabalho teve com objetivo o estudo da coagulação com sulfato de alumínio de águas preparadas para resultar cor verdadeira de $100 \mathrm{uH}$, com SH extraídas de turfa, de diferentes massas molares e diferentes porcentagens de ácido húmico e ácido fúlvico presentes.

\section{MATERIAIS E MÉTODOS}

\section{Extração e características das substâncias húmicas}

\section{Extração das SH utilizadas}

Para a preparação das águas de estudo foram utilizadas SH extraídas de turfa coletada junto às margens do rio MogiGuaçu, utilizando-se o seguinte procedimento (adaptado de Rosa et al, 2000):

· extração em solução $0,5 \mathrm{~mol} / \mathrm{L}$ de hidróxido de potássio $(\mathrm{KOH})$;

- tempo de agitação de 4 horas;
- razão turfa/extrator 1:20 (m/v); - temperatura ambiente $\left(22-27^{\circ} \mathrm{C}\right)$; - decantação pôr 48 horas;

- diálise até teste negativo para cloretos com $\mathrm{Ag} \mathrm{NO}_{3}$.

Fracionamento das $\mathrm{SH}$ em diferentes massas molares

As $\mathrm{SH}$, depois de extraídas, foram diluídas para concentração de $1,0 \mathrm{mg} \mathrm{mL}^{-1}$ e posteriormente filtradas. Inicialmente foi usado filtro de papel grosseiro $(3 \mu \mathrm{m})$ e depois membranas de $0,45 \mu \mathrm{m}$ com diâmetro de $90 \mathrm{~mm}$. A amostra foi fracionada nas faixas de massa molar $>100 \mathrm{kDa}, 30$ - $100 \mathrm{kDa},<30 \mathrm{kDa}$, utilizando-se o equipamento de Ultrafiltração (Vivaflow 50), equipado com bomba peristáltica e duas membranas em paralelo de polyethersulfone. Para o fracionamento em diferentes massas molares foi adotado o método de concentração da amostra (Duarte et al, 2001). A vazão máxima utilizada foi de até $300 \mathrm{~mL} \mathrm{~min}^{-1}$ com aproximadamente 1,5 atm de pressão.

Distribuição do carbono orgânico dissolvido (COD) na amostra de $\mathrm{SH}$, em relação às diferentes massas molares e percentagens de ácidos húmicos $(\mathrm{AH})$ e fulvicos (AF) presentes em cada fração

Para verificar a distribuição de diferentes massas molares na amostra de $\mathrm{SH}$, foi determinada a concentração de COD em cada fração após o fracionamento. Para isso, inicialmente foram filtrados $500 \mathrm{~mL}$ da amostra de SH extraída e diluída $\left(1 \mathrm{mg} \mathrm{mL}^{-1}\right)$ na membrana com poro de $0,45 \mu \mathrm{m}$ e mediu-se a concentração de COD. Fracionou-se, no equipamento de ultrafiltração, uma alíquota de $400 \mathrm{~mL}$ dessa amostra filtrada em membrana de $0,45 \mu \mathrm{m}$, nas diferentes massas molares (>100 kDa, $30-100 \mathrm{kDa}$ e $<30 \mathrm{kDa})$ e mediu-se o COD em cada fração. A medida do COD foi feita em um equipamento de carbono orgânico total TOC-V CPH/CPN/P da Shimadzu. Assim, por meio dos valores de COD, foi possível estimar a porcentagem de cada fração (>100 kDa, 30-100 kDa e $<30 \mathrm{kDa}$ ) de diferente massa molar na amostra de SH.

Para estimar a porcentagem de $\mathrm{AH}$ e AF em cada fração de diferente massa molar, foi utilizada a teoria que divide as $\mathrm{SH}$ segundo sua solubilidade, sendo: $\mathrm{AH}$ : solúvel em meioalcalino, masinsolúvel em meioácido; AF:solúvel tanto em meioácido comoem meio alcalino (Choudhry, 1984; Hayes etal, 1989). mento:

Foi adotado o seguinte procedi-

Coleta de $50 \mathrm{~mL}$ de cada fração de diferentes massas molares de $\mathrm{SH}$ : i) filtrado em membrana de $0,45 \mu \mathrm{m}$, ii) filtrado em membrana de $0,45 \mu \mathrm{m}$ e maiores de $100 \mathrm{kDa}$, iii) fração entre 30 e $100 \mathrm{kDa}$ e iv) fração menor do que $30 \mathrm{kDa}$;

- Realização de medidas de COD para cada fração;

- Acidificação com ácido clorídrico $\mathrm{PA}(\mathrm{HCl})$ até $\mathrm{pH} \leq 2$; por $24 \mathrm{~h}$;

Precipitação de AH e decantação

- Centrifugação das amostras em $4000 \mathrm{rpm}$ por 30 minutos;

- Coleta do sobrenadante para medidas de COD.

Assim, pela concentração de COD na amostra sem divisão em $\mathrm{AH}$ e $\mathrm{AF}$ e depois de acidificada, e precipitação dos $\mathrm{AH}$, tem-se no sobrenadante a concentração do $\mathrm{COD}$ que é devido à presença dos $\mathrm{AF}$. $\mathrm{A}$ concentração do COD correspondente à presença dos AH é, desta forma, obtida por diferença (Garcia et al, 1986): de $\mathrm{AH}$

COD total $-\mathrm{COD}$ de $\mathrm{AF}=\mathrm{COD}$

\section{Diagramas de coagulação}

Foram preparadas 4 águas de estudo, três com as fraçôes de diferentes massas molares $(>100 \mathrm{kDa}, 30-100 \mathrm{kDa}$ e $<30 \mathrm{kDa}$ ) e uma utilizando todas as frações juntas (filtrada em membrana de $0,45 \mu \mathrm{m}$ ). Cada água foi preparada com quantidade de $\mathrm{SH}$ suficiente para resultar em cor verdadeira de $100 \pm 5 \mathrm{uH}$, utilizando-se água de poço profundo. A temperatura em todos os ensaios foi mantida em $20^{\circ} \pm 1^{0}$. Foram feitas medidas de alcalinidade, condutividade e turbidez nas águas de estudo.

Para a confecção dos diagramas de coagulação foi variada a dosagem do coagulante sulfato de alumínio líquido que possuía concentração de 7,28 \% em massa de $\mathrm{Al}_{2} \mathrm{O}_{3}$ e massa específica igual a $1,304 \mathrm{~g} \mathrm{~L}^{-1}$ em relação a diferentes valores de $\mathrm{pH}$, para todas as águas de estudo. Para alterar o $\mathrm{pH}$ de coagulação foram utilizadas soluções $0,1 \mathrm{M}$ de ácido clorídrico $(\mathrm{HCl})$ e/ou de hidróxido de sódio $(\mathrm{NaOH})$. Todos os ensaios foram realizados em equipamento de jarteste, adotando-se os parâmetros físicos dos ensaios, apresentados na Tabela 1.

Foram feitas coletas de amostra após a mistura rápida para a medida do $\mathrm{pH}$ de coagulação, em todos os ensaios e para to- 
das águas de estudo. Após a mistura rápida e a floculação foram feitas coletas de amostras no tempo correspondente à velocidade de sedimentação de $0,5 \mathrm{~cm} \mathrm{~min}^{-1}$, para a leitura da cor aparente. Para as medidas de cor, foram utilizadas curvas de calibração adicionadas no espectofotômetro DR/4000 $\mathrm{U}$ da Hach, as quais foram construídas utilizando-se as SH extraídas. Os valores da cor foram obtidos por meio de uma curva padrão seguindo-se o procedimento descrito no"Standards Methods for the Examination of Water and Wastewater" (1998).

Assim, utilizando-se a curva padrão foi possível obter curvas para as $\mathrm{SH}$ nos valores de $\mathrm{pH}$ de 4,$0 ; 4,5 ; 5,0 ; 5,5 ; 6,0$; 6,5 e 7,0, através do procedimento de diluiçôes sucessivas. Com os valores das dosagens de coagulante e $\mathrm{pH}$ de coagulação, foram construídos os diagramas de coagulação, que contêm os valores de cor aparente remanescente.

\section{RESULTADOS E DISCUSSÁO}

\section{Distribuição do carbono orgânico dissolvido (COD) na amostra de SH, em relação as diferentes massas molares e as percentagens de ácidos húmicos (AH) e ácidos fulvicos (AF) presentes em cada fração}

Apesar de ainda não se ter uma teoria que seja consenso entre os pesquisadores que estudam $\mathrm{SH}$ a respeito do que seriam os AH e AF e a separação dos mesmos da fração húmica, a mais aceita $\mathrm{e}$ empregada é a que considera as diferentes solubilidades. Pelo emprego desta teoria, as frações de diferentes massas molares foram separadas em AH e AF. A Tabela 2 apresenta os valores de COD de cada fração de diferente massa molar obtida após o ultrafracionamento e os valores de COD após a separação em AH eAF. As Figuras 1 e 2 apresentam os valores em porcentagem.

Verifica-se na Figura 1 que a maior porcentagem do COD, após a amostra de $\mathrm{SH}$ ter sido filtrada em membrana de $0,45 \mu \mathrm{m}$ e fracionada em diferentes massas molares encontra-se na faixa de $0,45 \mu \mathrm{m}$ a $>100 \mathrm{kDa}$, com cerca de $75 \%$. Este fato demonstra que SH extraídas de turfa são formadas principalmente por $\mathrm{AH}$, os quais, possuem estruturas mais condensadas, com maiores números de agregados moleculares ligados e assim com massas molares que podem atingir centenas de milhares de daltons (Swift, 1985; Wershaw et al, 1985).

$\mathrm{Na}$ Figura 2 pode ser observado que há uma relação inversa da porcentagem de $\mathrm{AH}$ e AF com a diminuição da massa molar. Segundo a nova teoria proposta por Piccolo (2001) os AF seriam agrega- dos formados por pequenas moléculas constituídos em maior concentração por grupos oxigenados do que os $\mathrm{AH}$. Assim essas moléculas menores permaneceriam estáveis em solução pela repulsão de cargas negativas, devido à dissociação desses compostos oxigenados. Já os $\mathrm{AH}$, por apresentarem uma menor concentração

Tabela I - Parâmetros físicos adotados para os ensaios

\begin{tabular}{cc}
\hline Parâmetros físicos & Valores \\
\hline Tempo de mistura rápida (Tmr) & $5 \mathrm{~s}$ \\
Gradiente na mistura rápida $(\mathrm{Gmr})$ & $1000 \mathrm{~s}^{-1}$ \\
Tempo de floculação $(\mathrm{Tf})$ & $30 \mathrm{~min}$ \\
Gradiente de floculação $(\mathrm{Gf})$ & $15 \mathrm{~s}^{-1}$ \\
Velocidade de sedimentação $(\mathrm{V})$ & $0,5 \mathrm{~cm} \mathrm{~min}^{-1}$ \\
\hline
\end{tabular}

Tabela 2 - Concentração de COD nas frações de diferentes massas molares das $\mathrm{SH}$, sem fracionamento (total) e após fracionamento em $\mathrm{AH}$ e AF

\begin{tabular}{cccc}
\hline Fraçóes & \multicolumn{3}{c}{ COD $\left(\mathrm{mg} \mathrm{C} \mathrm{L}^{-1}\right)$} \\
& Total & AF & AH \\
\hline Filtrada em $0,45 \mu \mathrm{m}$ & 320,7 & 44,9 & 275,8 \\
Filtrada em $0,45 \mu \mathrm{m} \mathrm{e}>100 \mathrm{kDa}$ & 245,3 & 19,6 & 225,7 \\
30 a $100 \mathrm{kDa}$ & 38,22 & 27,87 & 10,35 \\
$<30 \mathrm{kDa}$ & 18,97 & 16,71 & 2,26 \\
\hline
\end{tabular}

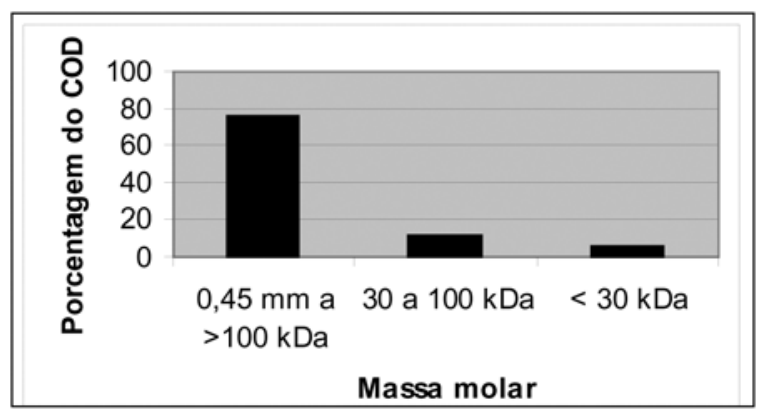

Figura I- Porcentagem do COD em relação a cada fração de diferente massa molar das $\mathrm{SH}$

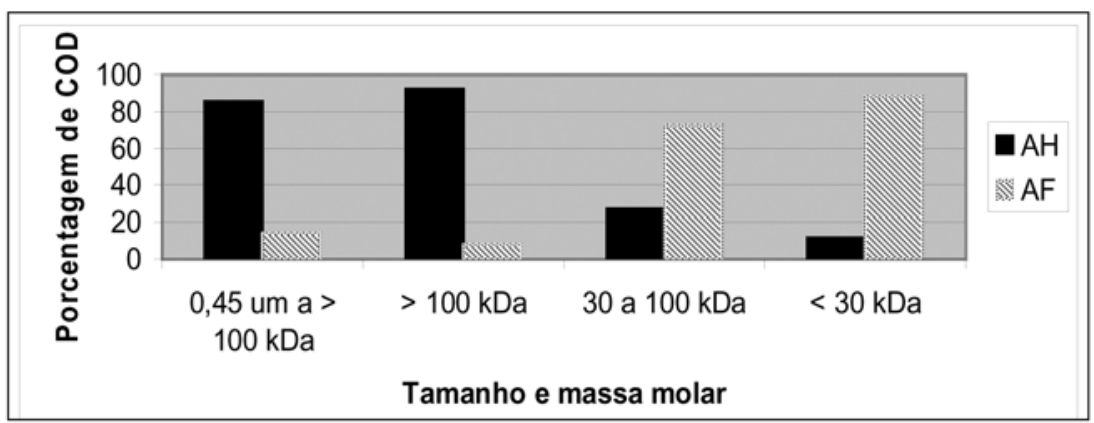

Figura 2- Porcentagem de COD nas frações de diferentes massas molares em relação a $\mathrm{AH}$ e $\mathrm{AF}$ 
de grupos oxigenados teriam maior força de atração entre as moléculas formando desta forma agregados maiores.

Burba et al, (1995) e Rocha et al, (1999) estudaram substâncias húmicas aquáticas (SHA) extraídas do rio Rhur na Alemanha e do Rio Negro no Brasil, respectivamente. Após separação das SHA em diferentes massas molares, utilizando um sistema seqüencial de ultrafiltração, os autores verificaram que a maior quantidade das SHA possuía massa molar entre 10 e $100 \mathrm{kDa}$. Geralmente cerca de 90 \% das SHA são constituídas de ácidos fulvicos aquáticos (AFA) e apenas 10\% correspondem aos ácidos húmicos aquáticos (AHA) (Malcom, 1985). Relacionando esses dados com os apresentados na Figura 2 observa-se que, como nas SHA, as frações com massa molar menor do que $100 \mathrm{kDa}$ apresentaram maior concentração de $\mathrm{AF}$ do que $\mathrm{AH}$.

\section{Diagramas de coagulação}

As Figuras 3, 4, 5 e 6 apresentam diagramas de coagulação representativos para as águas de estudo preparadas com as frações de diferentes massas molares $(0,45 \mu \mathrm{m}$ a $.100 \mathrm{kDa},>100 \mathrm{kDa}, 30 \mathrm{a}$ $100 \mathrm{kDae}<30 \mathrm{kDa}$ ), para $\mathrm{Vs}=0,5 \mathrm{~cm} \mathrm{~min}^{-1}$.

As $\mathrm{SH}$ são moléculas carregadas negativamente e podem ser removidas por dois mecanismos utilizando-se sulfato de alumínio como coagulante. Em uma regiāo de baixo $\mathrm{pH}(4 \mathrm{a} 5,5)$, as $\mathrm{SH}$ podem interagir com as cargas positivas do coagulante e formar um precipitado alumínio-humato. Em regiōes de $\mathrm{pH}$ mais alto (6 a 8$)$ as moléculas podem ser adsorvidas pelo hidróxido de alumínio formado em excesso e precipitarem.

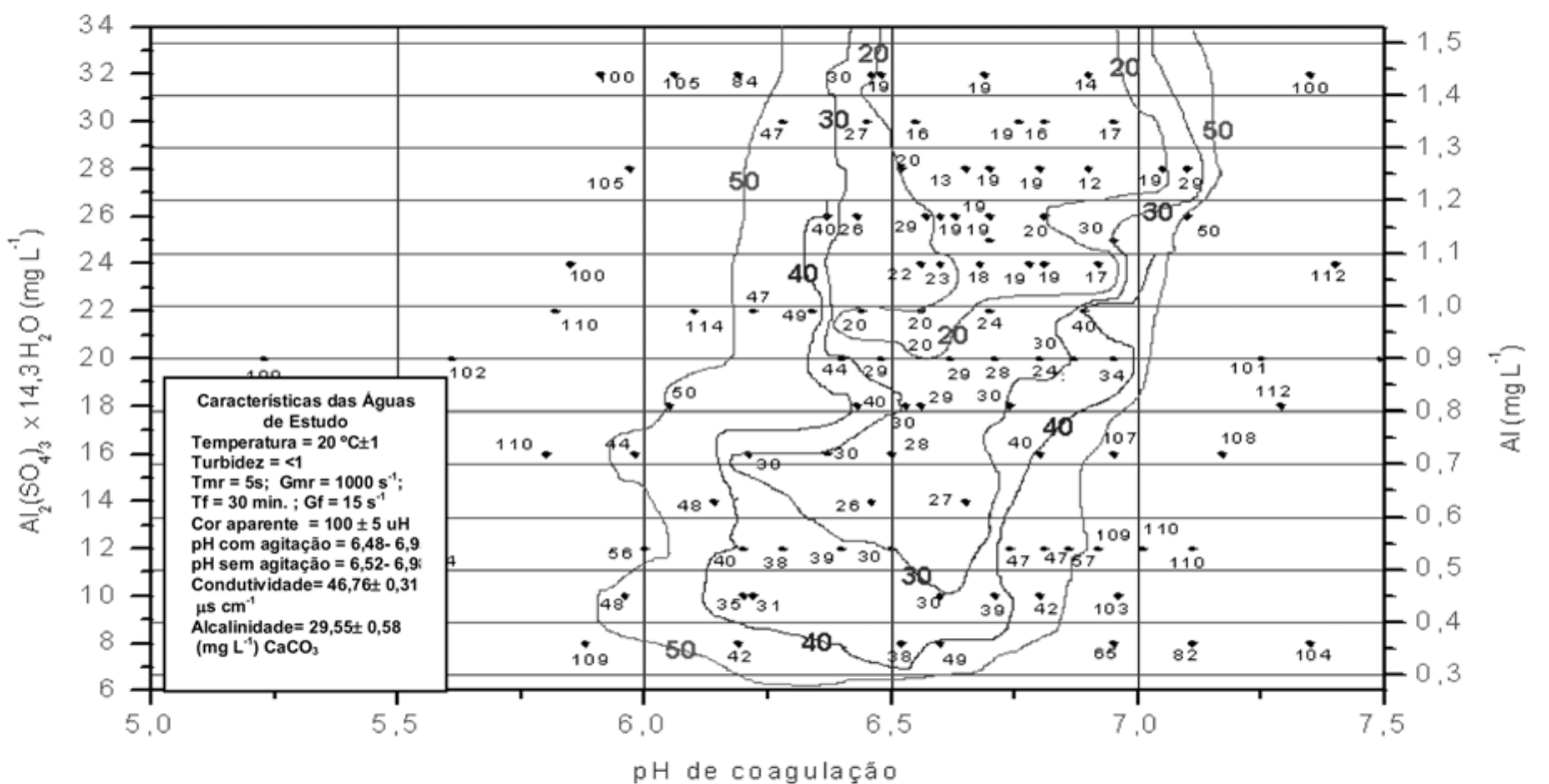

Figura 3 - Curvas com valores de cor aparente remanescente (uH), em função da dosagem de coagulante versus pH de coagulação para a água preparada com a fração de $\mathrm{SH}$ filtrada em membrana $0,45 \mu \mathrm{m}\left(V \mathrm{~s}=0,5 \mathrm{~cm} \mathrm{~min}^{-1}\right)$

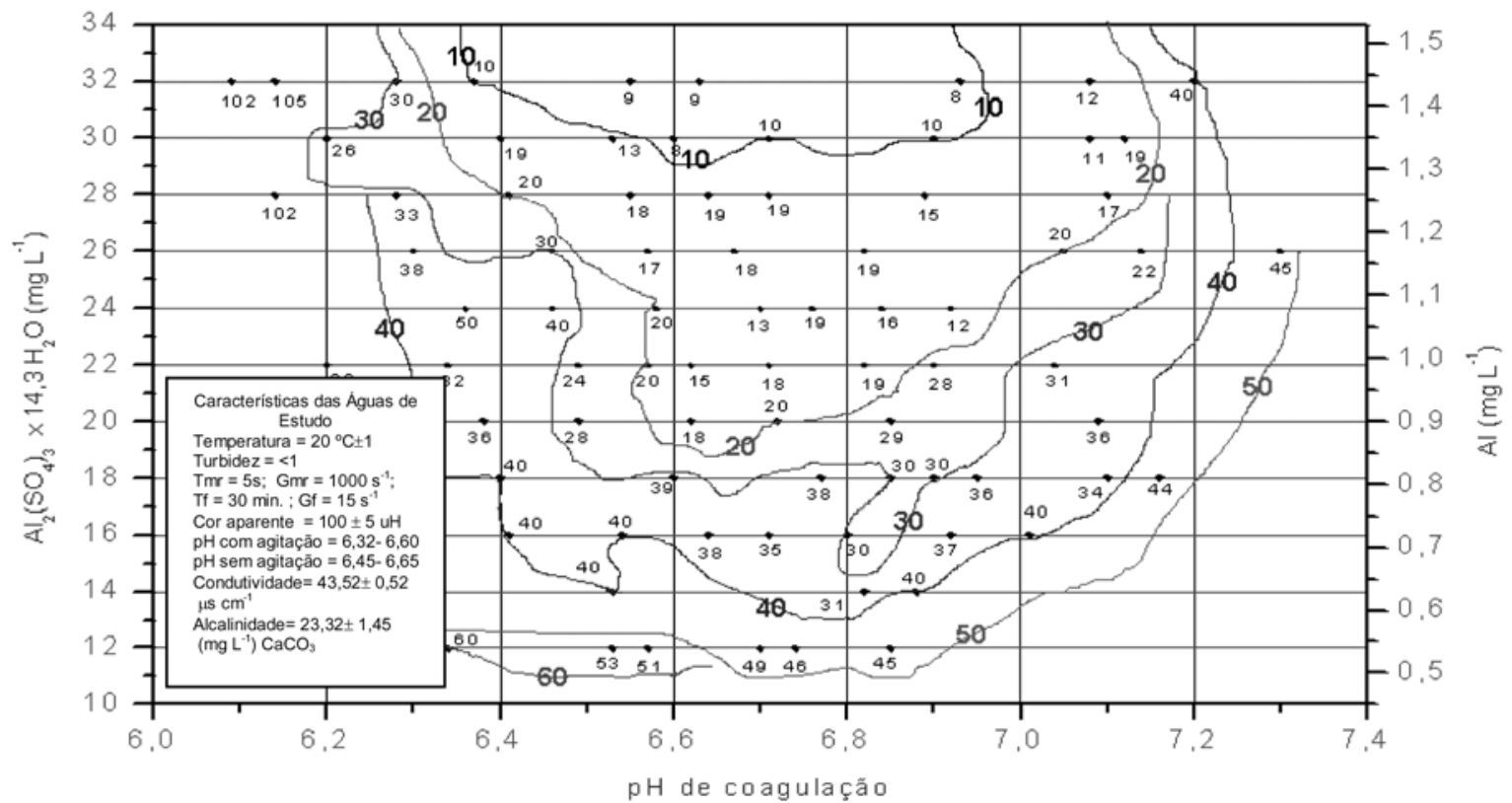

Figura 4 - Curvas com valores de cor aparente remanescente (uH), em função da dosagem de coagulante versus pH de coagulação para a água preparada com a fração filtrada em 0,45 $\mu \mathrm{m}$ e > $100 \mathrm{kDa}\left(\mathrm{Vs}=0,5 \mathrm{~cm} \mathrm{~min}^{-1}\right)$ 


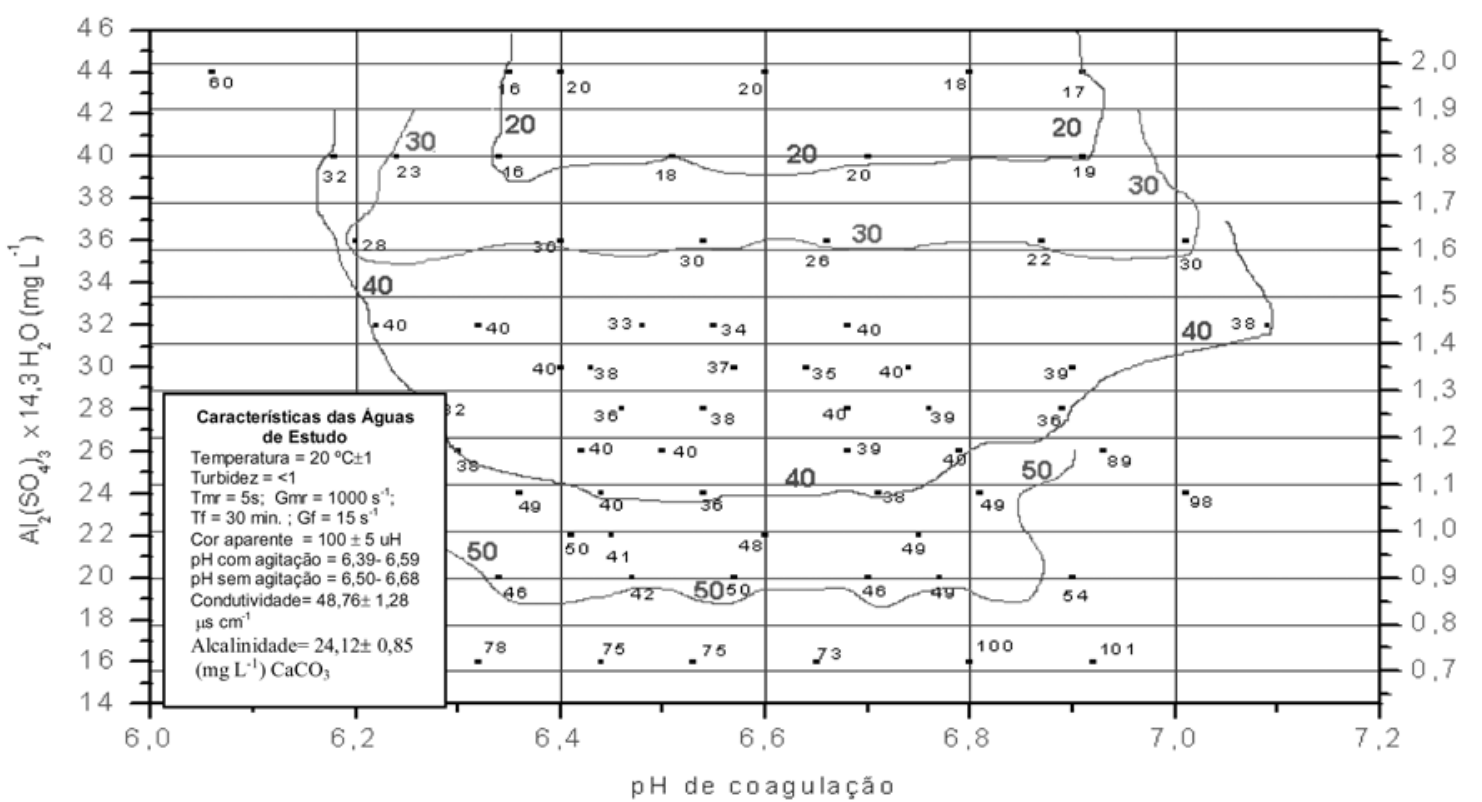

Figura 5 - Curvas com valores de cor aparente remanescente (uH) em função da dosagem de coagulante versus pH de coagulação para a água preparada com a fração 30 a $100 \mathrm{kDa}\left(\mathrm{Vs}=0,5 \mathrm{~cm} \mathrm{~min}^{-1}\right)$

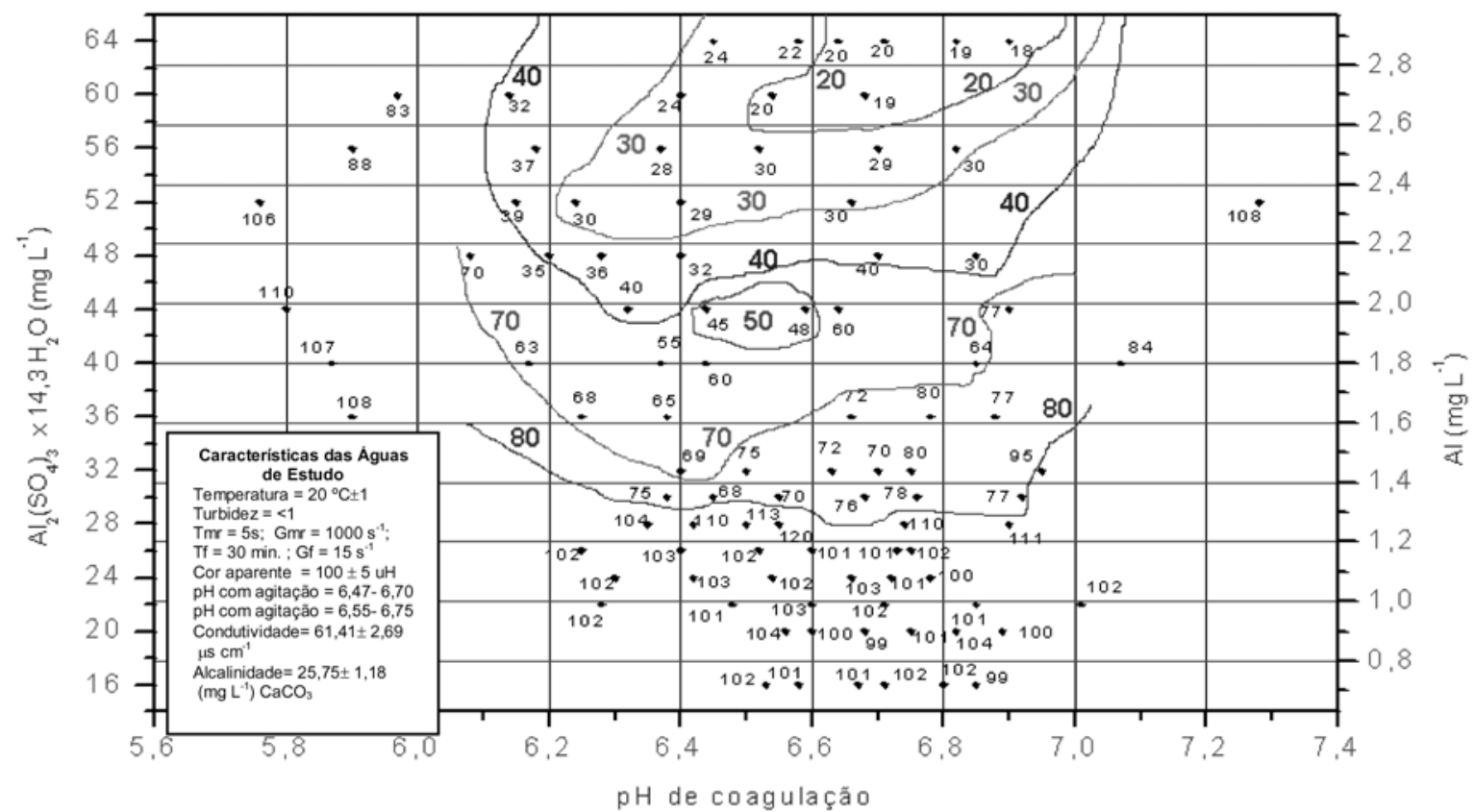

Figura 6 - Curvas com valores de cor aparente remanescente (uH) em função da dosagem de coagulante
versus pH de coagulação para a água preparada com a fração $<30 \mathrm{kDa}\left(\mathrm{Vs}=0,5 \mathrm{~cm} / \mathrm{min}^{-1}\right)$

Empiricamente é provado que quando se trabalha com água que possua cor alta e turbidez baixa, o $\mathrm{pH}$ de coagulação ideal é menor $(5,0$ a 6,0$)$ do que quando se tem uma água com turbidez alta $(7,0 \mathrm{a}$ 8,2 ), utilizando-se sulfato de alumínio como coagulante (Amirtharajah, 1989).

Por meio dos resultados apresentados nas Figuras 3, 4, 5 e 6 observa-se que independente da dosagem de coagulante, a melhor faixa de $\mathrm{pH}$ de remoção da cor aparente ficou entre 6,0 e 7,0. Verifica-se que nesta regiáo o mecanismo que impera é o de varredura, como proposto por Amirtharajah (1989). Nota-se nos diagramas de coagulação que a melhor região de remoção situou-se na faixa de $\mathrm{pH}$ entre 6 a 7, independente da massa molar. Para a água de estudo preparada com a fração de maior massa molar $(0,45 \mu \mathrm{m}$ a $>100 \mathrm{kDa})$, observou-se que as dosagens de coagulante utilizadas fo- ram iguais às empregadas para a água preparada contendo todas as frações juntas (filtrada em membrana de $0,45 \mu \mathrm{m}$ ). Este fato está de acordo com os resultados obtidos de COD, o qual mostrou que cerca de $75 \%$ do material filtrado pela membrana de $0,45 \mu \mathrm{m}$ é formado por moléculas com massa molar maior que $100 \mathrm{kDa}$. Entretanto, apesar dos valores das dosagens do coagulante utilizado terem sido iguais para essas duas águas, 
pode ser observado nos seus respectivos diagramas de coagulação (Figuras 3 e 4) que a massa molar teve influência nas curvas de cor aparente remanescente. Para a água preparada com moléculas de massa molar maiores que $100 \mathrm{kDa}$, foram obtidas curvas de cor aparente remanescente menores. Comparando-se os valores das dosagens de coagulante necessárias para a obtenção das curvas de cor aparente remanescente menor ou igual a $20 \mathrm{uH}$, verifica-se novamente a influência da massa molar. Enquanto para a água preparada com a fração de maior massa molar $(0,45 \mu \mathrm{m}$ a $>100 \mathrm{kDa})$ foi necessária uma dosagem de $20 \mathrm{mg} \mathrm{L}^{-1}$ para a obtenção de valores de cor remanescente menores ou iguais a $20 \mathrm{uH}$ (Figura 3), para a água preparada com a fração de menor massa $(<30 \mathrm{kDa})$ foi necessária uma dosagem de coagulante igual a $60 \mathrm{mg} \mathrm{L}^{-1}$ (Figura 6).

Relacionando esses resultados com os valores da porcentagem de $\mathrm{AH}$ e $\mathrm{AF}$ em cada fração, verifica-se que as águas de estudo preparadas com as fraçôes de menor massa molar (30 a $100 \mathrm{kDa}$ e $<30 \mathrm{kDa}$ ), que possuem maior concentração de $\mathrm{AF}$, necessitaram de uma maior dosagem de coagulante para que a coagulação, floculação e sedimentação resultassem eficientes.

\section{CONCLUSÕES E RECOMENDAÇÕ๊ES}

Com base no trabalho realizado, verificou-se que:

a) Substâncias húmicas de diferentes massas molares e diferentes porcentagens de ácidos húmicos e ácidos fúlvicos presentes, demonstram comportamento diferenciado nos diagramas de coagulação.

b)Quanto menor a massa molecular das moléculas de substâncias húmicas, maiores foram as quantidades de ácidos fúlvicos;

c) Quanto maior foi a porcentagem de ácidos fúlvicos presente nas fraçōes de substâncias húmicas de diferentes massas molares, empregadas para preparar a água, maior foi a dificuldade de sua remoção.

d) Somente com um estudo experimental similar ao realizado é possível estabelecer as condições de coagulação que conduzem à eficiência desejada.

Estes resultados foram importantes para melhorar o entendimento a respeito da remoção de cor de águas, o que hoje torna-se muito relevante. Pelo trabalho realizado, recomenda-se que jamais sejam extrapoladas as condições de coagulação de águas provenientes de fontes diferentes, mesmo que apresentem valores da mesma ordem de grandeza de cor verdadeira.

\section{AGRADECIMENTOS}

Os autores agradecem à FAPESP Fundação de Amparo à Pesquisa do Estado de São Paulo pela concessão de bolsa de doutorado ao primeiro autor e auxílio à pesquisa ao segundo autor.

\section{REFERÊNCIAS}

AIKEN, G. R. Isolation and concentration techniques for aquatic humic substances. In: HUMIC SUBSTANCES IN SOIL, SEDIMENT AND WATER: GEOCHEMISTRY, ISOLATION AND CHARACTERIZATION. 1ed. New York. Ed. John Wiley \& Sons. p. 363- 385.1985.

AMERICAN WATER WORKS ASSOCIATION (AWWA). Water quality and treatment: a handbook of community water supplies. 4. ed. 270p. 1990.

AMITHARAJAH, A. The mechanisms of coagulation. In: TECNOLOGIAS PARA TRATAMENTO DE ÁGUAS DE BASTECIMENTO, São Carlos, 20p. 1989.

AMIRTHARAJAH, A.; O'MELIA, C. R. Coagulation processes: Destabilization, mixing and flocculation. Water quality and treatment. 1 ed. Toronto. Ed. McGraw-Hill. 220p. 1990.

BURBA, P.; SHKINEV, V.; SPIVAKOV, B, Ya.. On-line fractionation and characterization of aquatic humic substances by means of sequencial-stage ultrafiltration. Fresenius J. Anal. Chem. v., 351, p.72-82, 1995.

CHOUDHRY, G. G. Humic substances: structural, photophysical, photochemical and free radical aspects and interactions with environmental chemicals. 1ed. New York Ed. Gordon and Breach Science. 154 p. 1995.

DI BERNARDO, L.; DI BERNARDO, A.; CENTURIONE FILHO, P. L. Ensaios de tratabilidade de água e dos residuos gerados em estaçôes de tratamento de água. 1ed. São Carlos. Ed. Rima, 237p. 2002.

DUARTE, M. B. O.; SANTOS, E. P. H.; DUARTE, A. P. Comparacion between diafiltration and concentration operation modes for the determination of permeation coefficients of humic substances through ultrafiltration membranes. Anal. Chim. Acta., v.442, p.155-164, 2001.

EDWARDS, G. A.; AMIRTHARAJAH, A. Removing color caused by humic acid. JAWWA, v. 77 , n. 3, p. $50-57$, March, 1985

GARCIA, D.; CEGARRA, J. ABAD, M. $A$ comparison between alkaline and decomplexing reagents to extract humic acids from low rank coals. Fuel Proc. Technol., v.48, p.51-60, 1986.

HAYES, M. H. B. et al. Humic substances II: in search of structure. 1ed. Chichester. Ed. John Wiley, 250p. 1986.

KIM, S. H.; MOON, B. H.; LEE, H. I. Effects of $\mathrm{pH}$ and dosage on pollutant removal and floc structure during coagulation. Microchemical Journal. v.68, p. 197-203, 2001.
ROCHA, J. C et al. Multi-method study on aquatic humic substances from the "Rio Negro" Amazonas state/Brazil. Emphasis on molecularsize classification of their metal contents. J. Braz. Chem. Soc., v. 10, p. 169-175, 1999.

ROSA, A. H.; ROCHA, J. C.; FURLAN, M. Substâncias húmicas de turfa: Estudo dos parâmetros que influenciam no processo de extração alcalina. Quim. Nova, v.23, p.472-476, 2000 .

Standards Methods for the Examination of Water and Wastewater. 20 ed. New Yok, APHA, AWWA, AWPCF. CD ROM. 1998.

SWIFT, R. S.. Fractionation of soil humic substances. In: HUMIC SUBSTANCES IN SOIL, SEDIMENT AND WATER: GEOCHEMISTRY, ISOLATION AND CHARACTERIZATION. 1ed. New York. Ed. John Wiley \& Sons, p. 387-408. 1985.

WERSHAW, R. L.,AIKEN, G. R. Molecular size and weight measurements of humic substance In: HUMIC SUBSTANCES IN SOIL, SEDIMENT AND WATER: GEOCHEMISTRY, ISOLATION AND CHARACTERIZATION. 1ed. New York. Ed. John Wiley \& Sons, p. 410-434. 1985.

Endereço para correspondência:

\author{
Luiz Di Bernardo \\ Escola de Engenharia de São \\ Carlos - USP \\ Departamento de Hidráulica e \\ Saneamento \\ Av. Trabalhador São-carlense, 400 \\ I3566-590 São Carlos - SP - Brasil \\ Tel: (I6) 3273-9528 \\ E-mail: bernardo@sc.usp.br
}

